[Agr. Biol. Chem., Vol. 30, No. 10, p. 967 972, 1966]

\title{
Studies on Amylases of Aspergillus oryzae Cultured on Rice
}

\author{
Part II. Some Properties of Glucoamylases \\ By Miyoko Ohga, Katsuzo Shimizu and Yuhei Morita \\ Research Institute for Food Science, Kyoto University, Kyoto \\ Received February 28, 1966
}

\begin{abstract}
Some physico-chemical and enzymatic properties of four fractions of glucoamylase, isolated and purified from a culture of Aspergillus oryzae on rice, have been investigated in order to characterize and differentiate the components. Although four fractions were homogeneous in sedimentation analysis, only two fractions were found to be homogeneous in electrophoresis. One of the components had a molecular weight of 69,000 and an isoelectric point of 3.3. Four components resembled one another in their properties, except minute differences in their electrophoretic mobilities, sedimentation coefficients and $\mathrm{pH}$-stabilities. These differences as well as those in the chromatographic behaviors suggest slight differences in the structures of the components. Nevertheless, the properties of these components, except the molecular dimension, were very similar to those of Taka-amylase B, which was isolated from Taka-diastase by Okazaki.
\end{abstract}

\section{INTRODUCTION}

In a previous paper, ${ }^{1}$ ) we demonstrated the isolation and purification of glucoamylase from a culture of Aspergillus oryzae on steamed rice, obtaining four fractions of glucoamylase. They were homogeneous in sedimentation analysis and identical or very similar in enzymatic properties but different from one another in chromatographic behaviors on ion-exchanger. All of these fractions could hydrolyze soluble starch by stepwise removal of glucose but their degrees of hydrolysis were about $60 \%$. Such properties were very similar to those of Takaamylase $B$ of Okazaki $^{2)}$ and Taka-amylase $B_{2}$ of Sawasaki, ${ }^{3)}$ which were isolated and purified from Taka-diastase.

In the present paper, we describe some physico-chemical and enzymatic properties of these glucoamylase fractions in order to characterize the enzymes and to differentiate them

1) Y. Morita, K. Shimizu, M. Ohga and T. Korenaga, This Journal, 30, 114 (1966).

2) H. Okazaki, J. Agr. Chem. Soc. Japan, 29, 181 (1955).

3) T. Sawasaki, Repts. Sci. Research Inst. (Tokyo), 36, $584,590(1960)$. from one another.

\section{MATERIAL AND METHODS}

Glucoamylases. Glucoamylases were isolated and purified from a culture of Aspergillus oryzae on steamed rice as described in the previous paper.1) Four fractions, A-1, A-2, A-3 and B-2, were used in this experiment. All of them were found to be homogeneous in sedimentation analysis, but were different from each other in chromatographic behaviors on ion-ex-" changer.

Electrophoresis. Electrophoretic experiments were performed with a Tiselius apparatus, Hitachi Model HT-A, equipped with a diagonal slit device of Svensson and a standard $11 \mathrm{ml}$ cell. The temperature of the water bath was kept at $0.6 \pm 0.05^{\circ} \mathrm{C}$. The protein solution was dialyzed in the cold for forty eight hours against buffer solutions. For photographing, Fuji process hard dry plates and a yellow filter were used. Mobilities, reduced to $0^{\circ} \mathrm{C}$, were calculated for the descending boundaries from measurements made on the plates by using the method described by Longsworth $^{4}$ and employing conductivities determined at $0^{\circ} \mathrm{C}$. The pHs of the solutions were measured with a Beckman $\mathrm{G}$ type $\mathrm{pH}$ meter. The adjustment of 4) L. G. Longsworth, J. Am. Chem. Soc., 65, 1755 (1943). 
constant ionic strength, 0.1 , of buffer solutions was achieved by adding sodium chlordide into $0.1 \mathrm{M}$ acetate buffer solutions.

Sedimentation and Diffusion. Ultracentrifugal studies were performed with a Spinco Model E ultracentrifuge, equipped with Schlieren optics and automatic rotor temperature control; in an acetate buffer solution of $\mathrm{pH} 5.0$ and ionic strength 0.1 adjusted by adding sodium chloride. In some experiments, a double sector cell was used to differentiate sedimentation velocities of different fractions. Making the usual corrections for temperature and viscosity, we evaluated sedimentation coefficients, $s_{20}, \mathrm{w}$, according to the method of Schachman. ${ }^{5}$

Diffusion were measured with a Tiselius apparatus, Hitachi Model HT-A, at $0.3^{\circ} \mathrm{C}$ in the same buffer as in the ultracentrifugal experiments. The photographing was performed by the same procedure as in the electrophoretic experiments. Diffusion constant, $D_{20}$, w, was calculated by using the maximum ordinate methods of Neurath ${ }^{6}$ ) and Longsworth ${ }^{7)}$ from measurements made on the plates by making the usual corrections for temperature and viscosity. Relative viscosity and density of the buffer solution were measured by means of an Ostwald viscometer and an Ostwald picnometer.

Molecular dimension was calculated with the intrinsic values of $s_{20, w}$ and $D_{20, w}$ extrapolated to zero concentration of the protein by assuming the partial specific volume as 0.75 .

Light Absorption. Ultraviolet absorption spectrum of the protein was measured by means of a Beckman Model DU spectrophotometer and a Hitachi EPS-2 recording spectrophotometer.

Measurement of Amylase Activity. Amylase activity was measured by the 3,5-dinitrosalicylate method as described in the previous paper. ${ }^{11}$

\section{RESULTS}

\section{Electrophoretic Behavior}

Boundary electrophoretic experiments were performed on about $1 \%$ solutions of four glucoamylase fractions, A-1, A-2, A-3 and B-2, in acetate buffer solution, $\mathrm{pH} 5.60$ and ionic strength 0.1. Electrophoretic patterns of four fractions at 180 minutes are shown in Fig. 1. A-1 exhibited three components, but the major

5) H. K. Schachman. in "Methods in Enzymology" edited by S. P. Colowick and N. O. Kaplan, Vol. IV, p. 55, Academic Press, New York, 1957.

6) H. Neurath, Chem. Revs., 30, 357 (1942)

7) L. G. Longsworth, J. Am. Chem. Soc., 69, 2510 (1947).

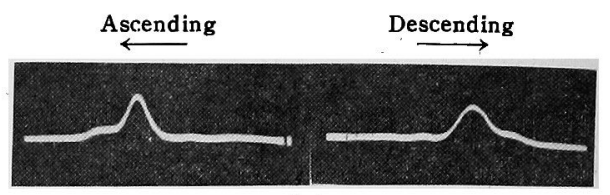

A-1

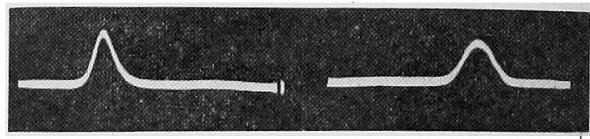

A-2

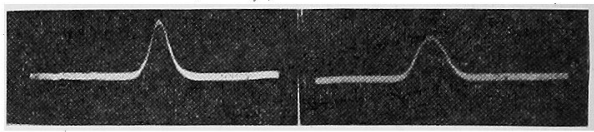

A-3

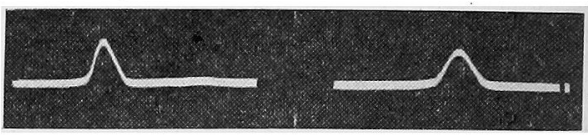

B-2

FIG. 1. Electrophoretic Patterns of Glucoamylase Fractions in Acetate Buffer, pH 5.6 and. Ionic Strength 0.1 , at $0.6^{\circ} \mathrm{C}$.

Protein concentration was $10 \mathrm{mg} / \mathrm{ml}$ in all cases. Photographs were taken at $10,800 \mathrm{sec}$, in a potential gradient 5.32 volts $\mathrm{cm}^{-1}$

one was found to be about $90 \%$ in the quantity. Fraction A-2 was almost homogeneous, but a little faster moving component, less than $5 \%$ in amount, was seen, whereas Fractions A-3 and B-2 were homogeneous under the expermental condition. Although A-3 exhibited a single peak in electrophoresis over the $\mathrm{pH}$ range between 4.1 and 5.6, two peaks appeared at $\mathrm{pH}$ 3.6. One of them seemed to be due to denaturation of A-3 component owing to such low $\mathrm{pH}$, because the recovery of the amylase activity at this $\mathrm{pH}$ was about 90\%, which fairly agreed with the quantity of the major peak. These facts lead to the conclusion that A-3 is homogeneous glucoamylase in electrophoresis. The pH-mobility curve of the component A-3 is shown in Fig. 2. By extrapolating the curve to zero mobility, the iso-electric point of $\mathrm{A}-3$ was found at $\mathrm{pH} 3.3$, showing that this enzyme was an acidic protein. Table I shows the mobilities of the major components of four fractions in acetate 


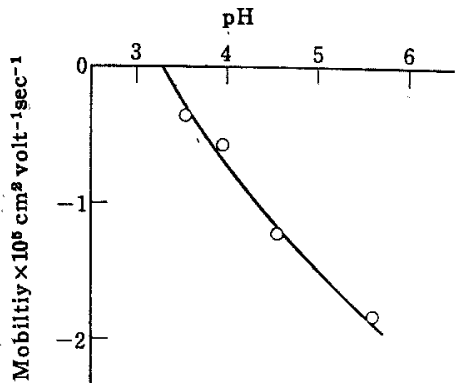

FIG. 2. pH-Mobility Curve of Glucoamylase Fraction A-3 in Acetate Buffer, Ionic Strength 0.1, at $0^{\circ} \mathrm{G}$.

Protein concentrations were $10 \mathrm{mg}$ per ml.

Table I. Electrophoretic Mobility aNd SedimeNTATION CONSTANT OF GLUCOAMYLASE COMPONENTS

\begin{tabular}{|c|c|c|c|}
\hline Fraction & & $\begin{array}{l}\text { horetic } \\
\text { ity* }\end{array}$ & $\begin{array}{l}\text { Sedimentation } \\
\text { constant }\end{array}$ \\
\hline A-1 & -2.2 & $\mathrm{~m}^{2} \mathrm{volt}^{-1} \mathrm{sec}^{-1}$ & $3.97 \mathrm{~S}$ \\
\hline A-2 & -2.6 & " & $4.32 \mathrm{~S}$ \\
\hline$A-3$ & -1.8 & $"$ & $3.91 \mathrm{~S}$ \\
\hline B-2 & -2.5 & $"$ & $4.05 \mathrm{~S}$ \\
\hline
\end{tabular}

* Measured on protein solutions, $10 \mathrm{mg} / \mathrm{ml}$, in acetate buffer, $\mathrm{pH}, 5.6$ and ionic strength 0.1 , and reduced to $0^{\circ} \mathrm{C}$. The values for A.1 and A-2 are those for the major com. ponents.

** Measured on protein solutions. $10 \mathrm{mg} / \mathrm{ml}$, in acetate buffer, $\mathrm{pH} 5.0$ and ionic strength 0.1 , and corrected to the standard condition, i.e., in water at $20^{\circ} \mathrm{C}$.

buffer, $\mathrm{pH} 5.6$ and ionic strength 0.1 , together with the sedimentation constants described later. Result shows that four major components are somewhat different from one another in the mobility, but such values of mobility but such value of mobility suggest that three other components than A-3 are also acidic proteins.

\section{Sedimentation and Diffusion}

All four glucoamylase fractions were homogeneous in sedimentation analysis in acetate buffer, $\mathrm{pH} 5.0$ and ionic strength 0.1 , the sedimentation patterns being presented in the previous paper. Their sedimentation coefficients for $1 \%$ protein concentration were calculated, as shown in Table I. The sedimentation coefficient of A-3 was measured for some different concentrations of the protein.

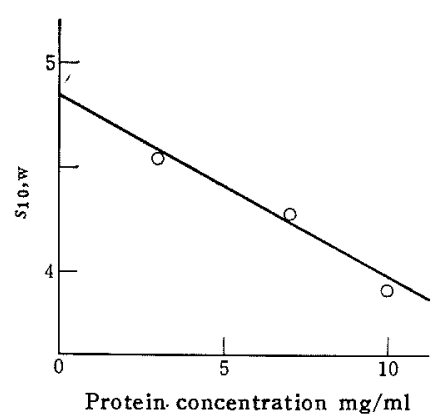

FIG. 3. Plot of Sedimentation Coefficient against Protein Concentration of Glucoamylase Fraction A-3.

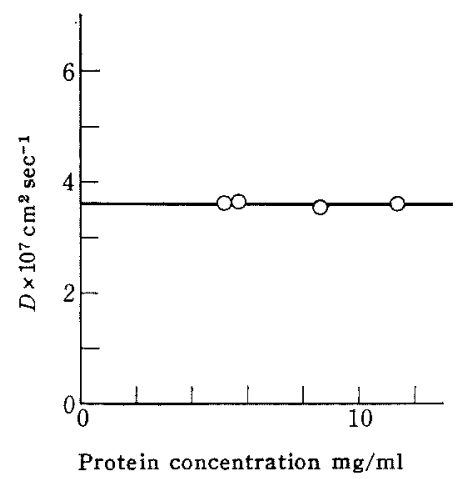

FIG. 4. Plot of Diffusion Constant against Protein

Concentration of Glucoamylase Fraction A-3.

Acetate buffer, $\mathrm{pH} 5.0$ and ionic strength 0.1 , at $0.3^{\circ} \mathrm{C}$.

Fig. 3 shows a plot of sedimentation coefficient versus the protein concentration. The extrapolated value to zero concentration was found to be $4.83 \mathrm{~S}$. On the same fraction, A-3, diffusion were measured by means of a Tiselius apparatus for some different concentrations of the protein. Fig. 4 shows a plot of diffusion constant in the buffer solution at $0.3^{\circ} \mathrm{C}$ versus the protein concentration, which gives the extrapolated value of $3.60 \times 10^{-7} \mathrm{~cm}^{2} \mathrm{sec}^{-1}$. Then the intrinsic diffusion constant under the standard condition, $D_{20, \mathrm{w}}$, was calculated at $6.93 \times 10^{-7} \mathrm{~cm}^{2} \mathrm{sec}^{-1}$. The molecular weight, calculated from the extrapolated values of the sedimentation coefficient and the diffusion con- 
stant, was found to be 69,000 by assuming the partial specific volume 0.75 . The fractional ratio was evaluated at 1.13. According to the equation of Perrin, ${ }^{8}$ the axial ratio was found to be 3.3 as a prolate or 3.4 as an oblate by assuming the molecular shape an ellipsoid. Because the other fractions were not homogeneous in electrophoresis or too small in amount to allows such detailed experiments, the molecular dimensions of the other components were not evaluated; but they would probably have similar values to those of the component A-3.

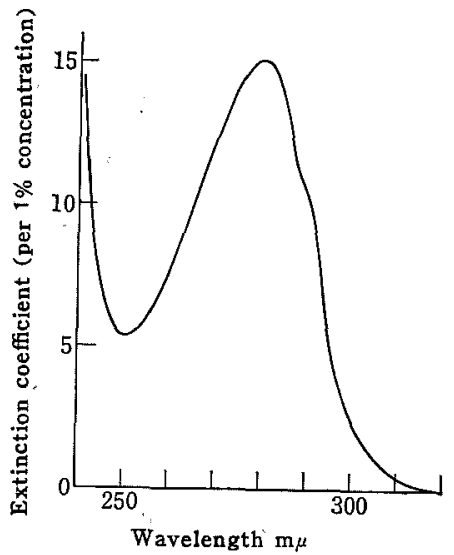

FIG. 5. Ultraviolet Absorption Curve of Glucoamylase Fraction B-2.

\section{Optical Property}

The ultraviolet absorption spectrum of gluco. amylase fraction B-2 is shown in Fig. 5. The spectrum was characteristic to a simple protein. The other fractions exhibited almost the same spectra, and the extinction coefficients at $280 \mathrm{~m} \mu$ per $1 \%$ concentration of the proteins were as follows: A-1, 12.46, A-2, 12.78, A-3, 12.94, and B-2, 14.98: The relative values seemed to correlate simply to the nitrogen or carbohydrate content of these preparations. In other words, there would be almost no differences in the tryptophan and tyrosine contents between four fractions on the protein basis excluding their carbohydrate moieties, as described in the succeeding paper.

\section{Enzymatic Properties}

a. pH-Activity relationship. The $\mathrm{pH}$-activity relationships of glucoamylase fractions were investigated as follows. One $\mathrm{ml}$ of $1 \%$ amylopectin, buffered with 0.025 m citrate-phosphate with varying $\mathrm{pH}$, was mixed with $1 \mathrm{ml}$ of enzyme solution. After the reaction mixture had been incubated for three minutes at $35^{\circ} \mathrm{C}$, the reducing sugar produced was determined by 3,5-dinitrosalicylate method as described in the previous paper. The $\mathrm{pH}$ activity curves thus obtained are shown in Fig. 6, which exhibits similar curves and the maximal activites in a $\mathrm{pH}$ range between 4.1
A-1

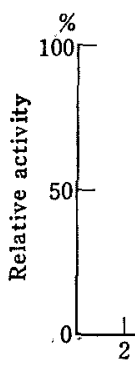

A-2
B-

A-3

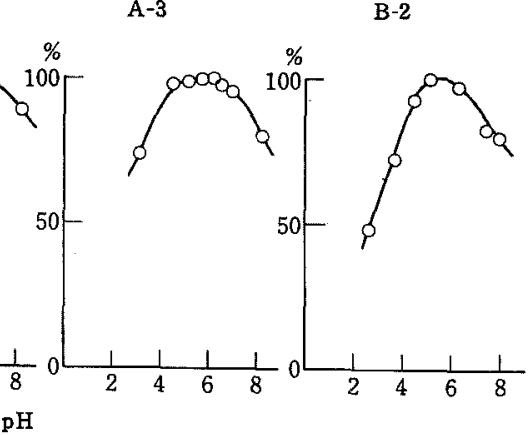

FIG. 6. pH-Activity Curves of Glucoamylase Fractions in Citrate-phosphate Buffer at $35^{\circ} \mathrm{C}$, Determined with $1 \%$ Amylopectin by 3,5-Dinitrosalicylate Method.

See text.

8) F. Perrin, J. Phys. radium, 7, 1 (1936). 
A-1

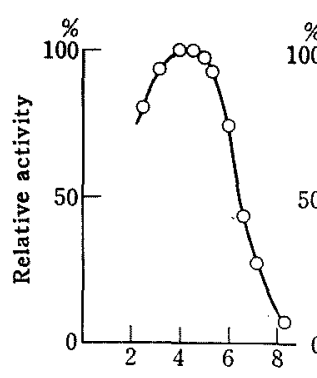

$A-2$

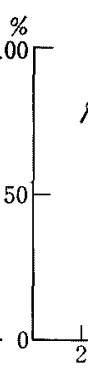

A-3

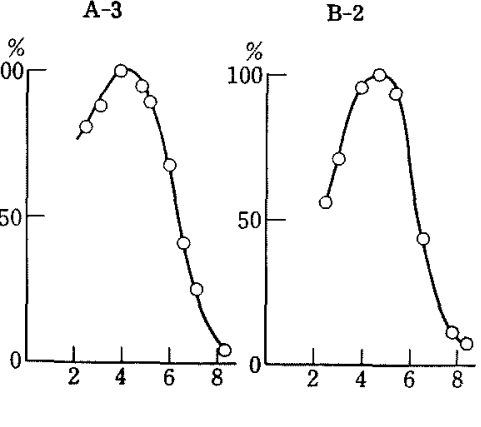

FIG. 7. pH-Stability Curves of Glucoamylase Fractions in Citrate-phosphate Buffer at $40^{\circ} \mathrm{C}$.

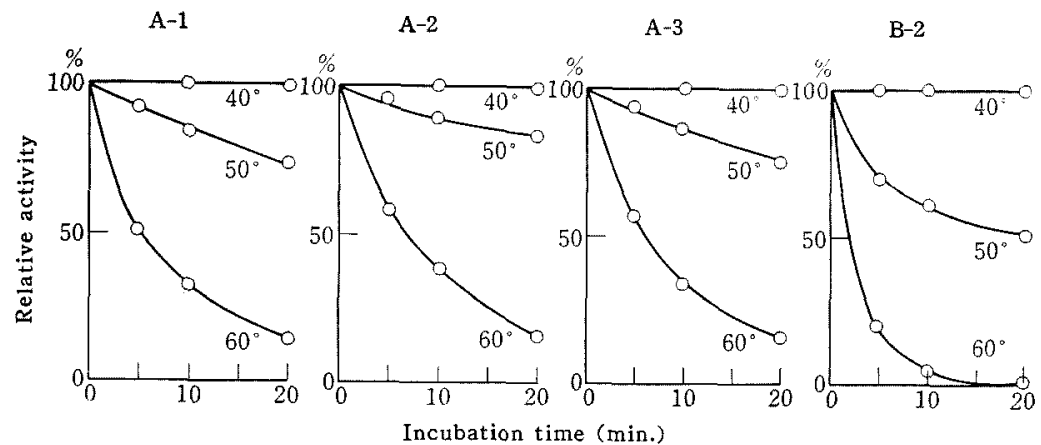

FIG. 8. Thermo-stability of Glucoamylase Fractions in Citrate-phosphate Buffer, pH 5.4.

Two $\mathrm{mg}$ of enzyme was incubated in $2 \mathrm{ml}$ of buffer solution. Aliquots, $0.5 \mathrm{ml}$, were analyzed by 3.5-dinitrosalicylate method after incubation.

nd 4.7. These curves were very similar to aat of Taka-amylase $\mathrm{B}^{21}$ or those of glucomylases from Rhizopus delemer ${ }^{9}$ and from Lspergillus niger. ${ }^{10\}}$ This fact suggests that all $f$ four fractions as well as the other glucomylases should have the same essential ionizd groups.

b. pH-Stability. The pH-stability relationlips of four glucoamylase fractions were lucidated as follows. The enzyme, about $\mathrm{mg}$, dissolved in $2 \mathrm{ml}$ of $0.013 \mathrm{M}$ citratehosphate buffer, $\mathrm{pH} 3.2$ to 8.2 , as incubated or thirty minutes at $4^{\circ} \mathrm{C}$, and then the survivIg glucoamylase activities were determined

9) L. L. Phillips and M. L. Caldwell, J.Am. Chem. Soc., 3,3559 (1951).

10) S. A. Barker and J. G. Fleetwood, J. Chem. Soc., 1957, at $\mathrm{pH} 4.8$ by 3,5-dinitrosalicylate method. The pH-stability curves are shown in Fig. 7, which indicates that four glucoamylases were most stable in a $\mathrm{pH}$ range of 5.2 to 6.2 . These behaviors were similar to that of Taka-amylase B. ${ }^{2)}$

c. Thermo-stability. After four glucoamylase fractions had been incubated in $0.013 \mathrm{~m}$ citrate-phosphate buffer, $\mathrm{pH}$ 5.4, at 40,50 and $60^{\circ} \mathrm{C}$ for 5,10 and 20 minutes aliquots were analyzed for surviving activities. Fig. 8 indicates that three fractions except B-2 show almost the same stabilities. These behaviors were also very similar to that of Taka-amylase B. ${ }^{2)}$ The heat inactivation of glucoamylases was rather promoted by the presence of calcium ions, unlike $\alpha$-amylases. Thus, Fraction 
A-3 still exhibited $15.3 \%$ of the original activity after incubation for twenty minutes at $60^{\circ} \mathrm{C}$ in the absence of calcium ions, whereas it exhibited only $4.7 \%$ activity under the same condition in the presence of $0.02 \mathrm{M}$ calcium chloride. The other fractions exhibited almost the same behavior. These negative effects of calcium ion was also observed on the heatdenaturation of glucoamylase from Rhizopus delemer. ${ }^{10)}$

\section{DISCUSSION}

In the present experiments, some physicochemical and enzymatic properties of four glucoamylase fractions of Aspergillus oryzae have been investigated. Unlike to demonstration on Taka-amylase B by Okazaki, ${ }^{2)}$ the glucoamylase of Aspergillus oryzae has a molecular weight of 69,000 , which is rather similar to that of glucoamylase prepared from Rhizopus delemer by Fukumoto et al. ${ }^{11)}$ As will be described in our succeeding paper, the glucoamylase of Aspergillus oryzae contains about $30 \%$ carbohydrate in dry basis, so the protein moiety will have a molecular weight of 50,000. Although the ultracentrifugal studies showed that all of these fractions were homogeneous, the electrophoretic experiments revealed that two fractions, A-1 and A-2, were not yet homogeneous. Such heterogeneity should prevent the absolute discussion on the differentiation of four glucoamylase components, but the differencés between them in electrophoretic and sedimentation behaviors under the same condition suggest slight differences in the structures of these components. Especially, the component B-2 differed originally in the fractionation steps from the other three components, so this component enzymatically had somewhat different properties, i. e., heat- or pH-stability, although other enzymatic properties were

\footnotetext{
11) J. Fukumoto et al., unpublished. See S. Ono, K. Hiromi
} and M. Jinbo, J. Biochem. (Tokyo), 55, 315 (1964). almost the same. These tacts seemed to con relate to the differences in the structure of the enzyme, especially the contents of carbo hydrate, which would affect on the physice chemical properties but not on the enzymatic reaction such as the $\mathrm{pH}$-activity relationshir or the mode of reaction. ${ }^{1)}$ High solubilitie: in concentrated ammonium sulfate solution also seemed to correlate to the high content: of carbohydrate, and this behavior prevents the enzyme from being salted out. In connection with this, much effort to crystallize the enzyme with organic solvents was also unsuccessful, because the enzyme was very unstable by the addition of organic solvents even in the presence of calcium or other divalent cations at low temperature. This was in the same situation as' that of Taka-amylase $\mathrm{B}^{2}{ }^{2}$ According to the Perlman's calculation method ${ }^{12 !}$ based upon the electrophoretic mobilities and molecular dimensions, four glucoamylase components should differ from one another in only one or two net charges in the molecules. However, true difference in these isozymes should not be elucidated until the compositions and the structures are clarified. In addition, all of four glucoamylases were acidic proteins, the isoelectric points being about $\mathrm{pH} 3.3$, although the values of electrophoretic mobilities did not always correspond to the order of the elution from the anion-exchanger described in the previous paper. The glucoamylases from Aspergillus oryzae cultured on rice anyway had very similar properties to those of Taka-amy: lase B except their molecular dimensions.

Acknowledgement. The authors wish to express their thanks to Dr. T. Ando, Mr. K. Kuriyama and Mr. S. Imayasu, Okura Shuzo Co., Kyoto, for their kind advice and discuse sions. Thanks are also due to Miss Nobuke Kada for her helpful assistance.

12) G. E. Perlman, J. Am. Chem. Soc., 71, 1146 (1949), 